\title{
Demonstrativer Konsum im Social Web. Über neue Sozialfiguren, ihren Umgang mit Produkten und dessen gesellschaftspolitische Konsequenzen
}

\section{Zusammenfassung}

Der Beitrag widmet sich der Frage, wie die Sozialen Medien nicht nur das Design und Marketing von Marken und Produkten verändern, sondern wie dadurch auch neue Sozialfiguren entstehen. ${ }^{15}$ Sie nutzen Konsumprodukte jeweils explizit als Medien, um Bekenntnisse zu ästhetischen, vor allem aber politischmoralischen Werten abzulegen und damit zugleich Individualität zu bekunden. Erhalten Produkte damit zusätzliche Funktionen, so kommt es dadurch aber auch zu neuen sozialen wie ökologischen Herausforderungen.

Individualisierter Konsum bedeutet in den heutigen westlichen Wohlstandsgesellschaften nicht mehr nur, sich durch materielle Statussymbole, die Vorliebe für bestimmte Marken oder den Mix einzelner Lifestyles von anderen abzugrenzen. In einer Gesellschaft, in der die materiellen Bedürfnisse weitgehend befriedigt sind, gilt vielmehr, frei nach Brecht, dass nach dem Fressen irgendwann auch die Moral kommt. Dann werden Werte zu einem entscheidenden Distinktionssymbol. Sie aber manifestieren sich ihrerseits nirgendwo besser als in Konsumprodukten. Diese fungieren dann als Bekenntnismedien; mit ihnen kann man sich als verantwortungsvoll, moralisch sensibel und sozial engagiert in Szene setzen.

Doch noch aus einem zweiten, grundlegenderen Motiv heraus führt der Wohlstand zu einer moralisch-politischen Aufladung des Konsums. Wenn nämlich einerseits mehr denn je konsumiert wird, dann wird andererseits auch ein alter Vorbehalt gegen Konsum umso virulenter. Dieser Vorbehalt besteht darin, dass man befürchtet, Konsum sei ,an sich' etwas Sündiges und Triebhaftes, bedeute Verschwendung und Raubbau, ja sei egoistisch und asozial. Damit gilt er als das Gegenteil zur Produktion, zur Arbeit, zum Fleiß, zu allem Sinnvollen und Vernünftigen. Sowohl in der Religion, vielleicht am stärksten im Protestantismus, aber auch in diversen Weltanschauungen, nicht nur im Sozialismus, wurde und wird die Arbeit sogar verklärt und überhöht, so dass der Konsum

15 Der Text ist unter Verwendung der männlichen Form für Berufs- und Personengruppen und -bezeichnungen verfasst Gemeint sind Personen aller Geschlechteridentität gleichermaßen. 
umso mehr als Negativbild, als schlechter Gegenpol erscheint. Um die in einer Wohlstandsgesellschaft mehr denn je konsumierenden Menschen mit ihrem Konsum zu versöhnen, muss dieser also entweder selbst als Arbeit verstanden werden oder aber moralisch qualifiziert werden können. Beides findet in der heutigen Gesellschaft statt.

Das Marketing hat es geschafft, dass der Konsum vielfältig als Arbeit erfahren wird (vgl. Ullrich 2018), doch das Marketing entwickelt genauso Geschäftsmodelle, die darauf angelegt sind, den Konsumenten das Gefühl zu geben, sie würden allein schon mit ihren Konsumentscheidungen dazu beitragen, die Welt ein bisschen besser zu machen. So erklärt man den Konsumenten, wie nachhaltig oder wie sozial der Entstehungsprozess eines Produkts ist oder dass es den Bedingungen von Fair-Trade entspricht oder dass ein Teil des Kaufpreises für ein ökologisches, ein medizinisches, ein gewerkschaftliches Projekt verwendet wird. Damit lässt sich das schlechte Gewissen kompensieren, das mit dem Konsum traditionell verbunden ist.

Der eigene Wohlstand, der zuerst nur schlechtes Gewissen forciert, wird also dazu verwendet, gutes Gewissen zu bereiten. Das aber führt zu der paradoxen Folge, dass letztlich oft sogar noch mehr konsumiert wird: Je mehr jemand etwas konsumiert, das schlechtes Gewissen bereitet, desto mehr wird diese Person zudem konsumieren, um sich vom schlechten Gewissen wieder zu befreien. Und wenn diese Person erleichtert ist, die eigenen Schuldgefühle überwinden zu können, es gar genießt, sich als verantwortungsvoll, als engagiert zu erleben, entsteht der Impuls, das auch anderen mitzuteilen.

Daher werden gerade die Sozialen Medien zu einem Ort von Bekundungen guten Gewissens, zum Ort von Wertbekenntnissen. Und der Wunsch, vielleicht sogar der Druck, auf Plattformen wie Facebook, Instagram oder YouTube regelmäßig präsent zu sein, kann den Konsum von Produkten, mit denen man sich als moralisch integer oder als gesellschaftlich engagiert zu präsentieren vermag, sogar noch weiter ankurbeln. Diese Produkte haben also sowohl eine therapeutische als auch eine repräsentative Funktion: Sie sollen gutes Gewissen bereiten, aber auch so verfasst sein, dass sich Bekenntnisse zu Werten mit ihnen eindrucksvoll in Szene setzen lassen. Idealerweise handelt es sich somit um Produkte, die nicht nur moralisch qualifizieren, sondern die auch fotogen sind: ästhetisch interessant und anspruchsvoll, voller Distinktionskraft. Dann können sie das Bekenntnis, das jemand ablegt, mit der Autorität von Stil und Geschmack unterstreichen. Wer sich mit diesen Produkten umgibt, kann sich dann als gleichermaßen verantwortungsbewusst und stilsicher, engagiert und cool, moralisch und ästhetisch präsentieren.

Menschen, die ästhetisch und moralisch reflektiert konsumieren und damit in zweifacher Hinsicht Individualität ausprägen, könnte man als Konsumbürger bezeichnen. Sie treten die Nachfolge der Bildungsbürger an, die lange Zeit eine 
gesellschaftliche Vorbildfunktion einnahmen, ästhetisch sowie moralisch Standards setzten sowie den Kanon der Hochkultur bestimmten. Entsprechend definieren heute Konsumbürger den Kanon der Konsumkultur und das, was über Konsum verhandelt wird. Und wie sich in der Geschichte der Bildungsbürger einige mehr für Kunst als für Politik interessierten, andere hingegen eher emanzipatorische Ziele vertraten, so beanspruchen unter den heutigen Konsumbürgern einige vor allem ästhetische Stilsicherheit, während andere ihre Aufgabe stärker darin sehen, sich politisch zu engagieren.

Diejenigen, die sich auf Stil und Geschmack konzentrieren, werden häufig als Hipster bezeichnet, die anderen sind entsprechend als Aktivisten oder Moralisten zu definieren. Und sofern sie mit ihren Konsumentscheidungen jeweils andere beeinflussen und als Vorbilder fungieren, gehören beide zu den ,Influencern'. Das alles sind neue Sozialfiguren, also Typen, die für die Gesellschaft insgesamt eine prägende, orientierende Rolle spielen (vgl. Moser/Schlechtriemen 2018).

Zahlenmäßig sind Influencer nur eine Minderheit, aber mit der Wirkung, die sie mit ihrem Verhalten haben, erreichen sie sehr viele Menschen. Generell kann man sagen, dass Sozialfiguren jeweils typisch für eine Zeit sind; durch sie entwickeln sich auch neue Institutionen oder neue Formate. So sind durch die verschiedenen Spielarten von Konsumbürgern innerhalb weniger Jahre zahlreiche neue Marken, auch neue Produkttypen entstanden, die an die Bedingungen individualisierten Digitalkonsums angepasst sind. Es sind jeweils Produkte, die vordergründig noch eine traditionelle Funktion - früher hätte man gesagt: einen Gebrauchswert - haben, welche aber vor allem als Anregung dafür dient, wie man sich damit inszeniert. Nicht dass man sich vor Nacktheit schützt oder warm angezogen ist, führt dann zum Kauf eines Kleidungsstücks, sondern man konsumiert es, weil man damit besser als mit Anderem Stil, Moral, Coolness oder eine emanzipiert kritische Gesinnung unter Beweis stellen kann.

Auch etablierte Marken nehmen sich gesellschaftlich relevanter Themen an und bringen etwa T-Shirts mit einem Slogan - Motto-Shirts - auf den Markt: Ende 2016 hat Dior ein T-Shirt mit dem Slogan "We should all be feminists" auf den Markt gebracht - dies ein Zitat eines berühmten Buchtitels der nigerianischen Schriftstellerin Chimamanda Ngozi Adichie. Über Nacht wurde dieses Shirt Kultobjekt, nachdem Rihanna - als eine der weltweit erfolgreichsten Influencerinnen - es auf ihrem Instagram-Account gepostet hatte. 


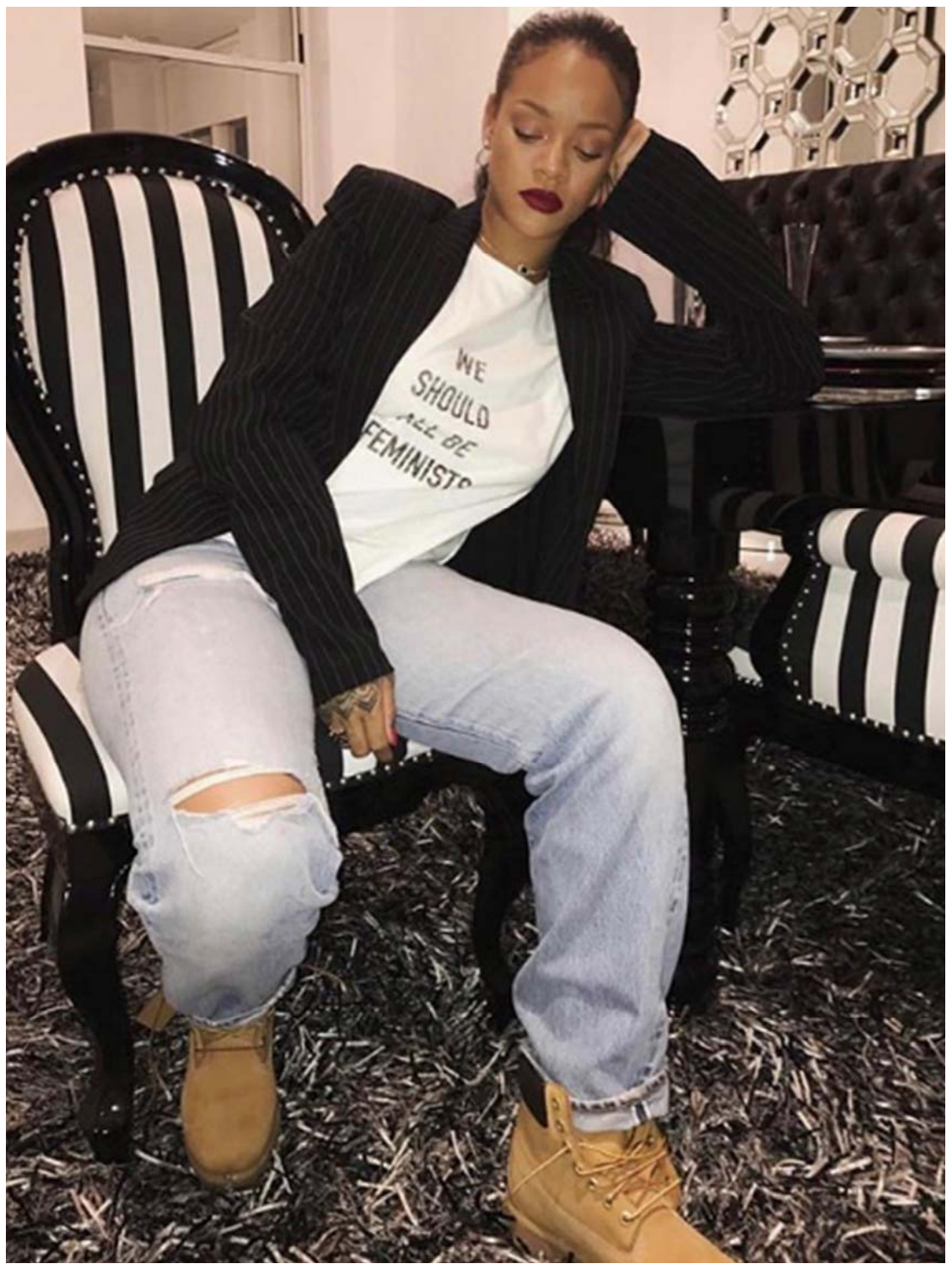

Abb. 1: Rihanna postet das Motto-Shirt von Dior auf Instagram

Quelle: https://www.instagram.com (oc) Er PRAeb8/ 
Daraufhin haben sich andere Stars auch mit diesem T-Shirt gezeigt. Eine wichtige Rolle spielte es beim ,Women`s March', der im Januar 2017 nach der Amtseinführung von Donald Trump stattfand, gegen dessen Wahlsieg der Slogan nicht zuletzt auch gerichtet war. Aber auch bei Modebloggern und Influencern war dieses T-Shirt beliebt; so inszenierte sich zum Beispiel Chiara Ferragni, die erfolgreichste Influencerin Italiens mit über 15 Millionen Followern, mit dem Shirt. Da ein Motto-Shirt leicht nachzumachen ist, hat allerdings kaum jemand das Original für 710 Dollar gekauft, sondern sich lieber eine der zahlreichen Adaptionen besorgt, die als Bekenntnismedium nicht minder geeignet sind. (Nur wer Stil oder Exklusivität unter Beweis stellen will, darf sich auf keine bloße Reproduktion einlassen, außer es handelt sich um eine besonders witzige Variante, die ihrerseits über hohen Distinktionswert verfügt.)

Allerdings stellt sich die Frage, wie überzeugend eine Marke wie Dior einen feministischen Slogan vertreten kann: Ist die Marke nicht mit einem ganz anderen Frauenbild groß geworden? Ist dieses Frauenbild hinreichend emanzipiert, damit ein feministisches Bekenntnis glaubhaft ist? Der Vorwurf des Opportunismus oder einer gewissen Leichtfertigkeit steht hier im Raum - und er betrifft auch nicht nur die Marke, sondern ebenso viele, die ein solches T-Shirt tragen oder kopieren und einfach mal ein Bild bei Instagram davon posten.

Für ein seriöses Bekenntnis ist es sicher glaubwürdiger, Produkte von Marken zu kaufen, die eine einschlägige Gründungsgeschichte und Agenda haben. Bezogen auf feministische Anliegen wäre etwa an Wildfang zu denken, die vor fünf Jahren gegründet wurde - ein eindeutiges feministisches Projekt in der gesamten Organisation der Abläufe, anders aufgebaut als herkömmliche Unternehmen; zudem werden Teile der Erlöse für Projekte verwendet, die Frauen zugutekommen. Auch Wildfang brachte ein Motto-Shirt auf den Markt. Es trägt den Slogan „Wild feminist“, und man findet es auf Instagram-Accounts ebenfalls viele hundert Mal, sicher nicht zuletzt deshalb, weil sich ein solcher Slogan genauso ironisch und mit einem Augenzwinkern wie auch ernst und kämpferisch inszenieren lässt. 


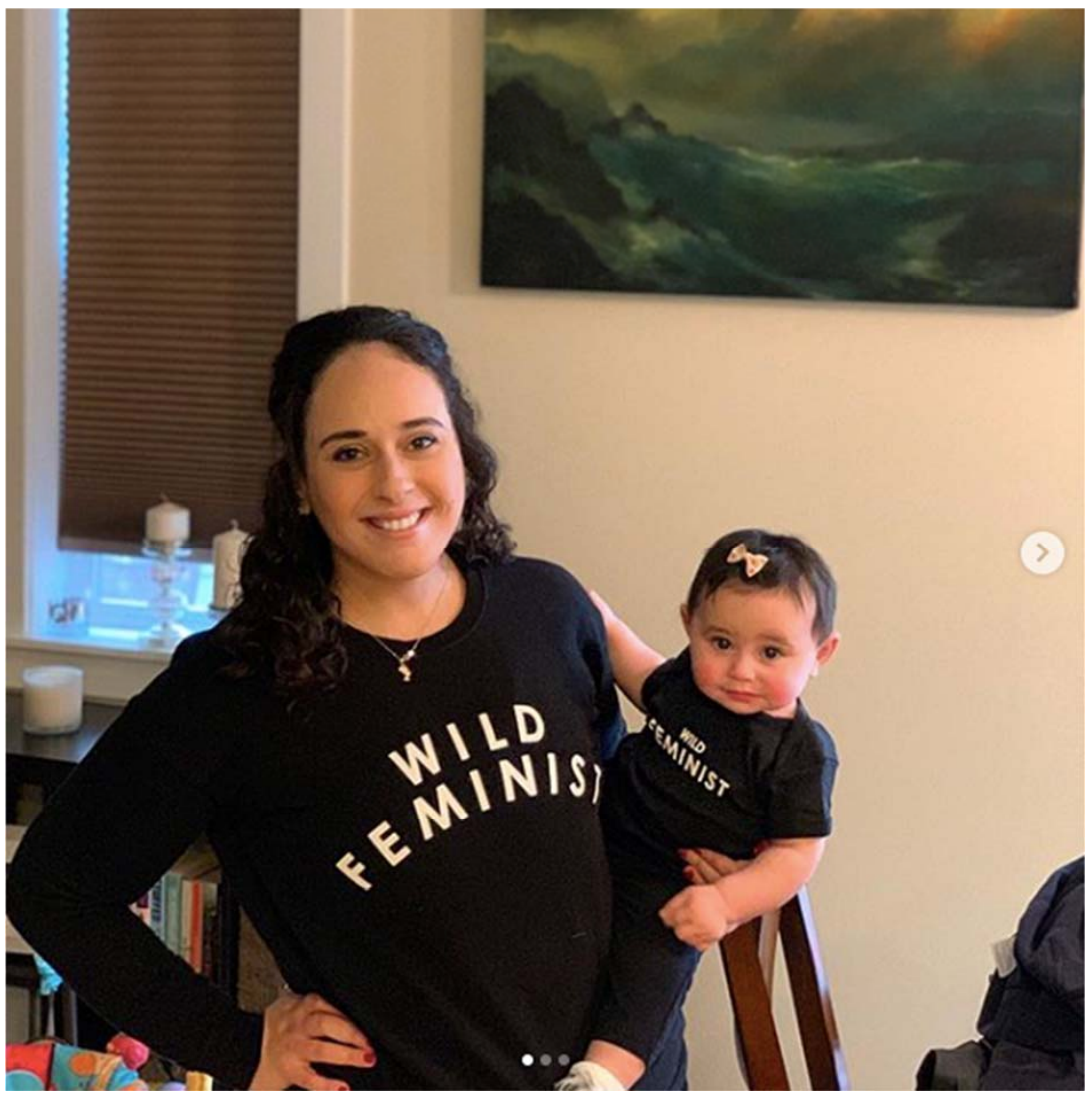

Abb. 2: Eine Konsumentin präsentiert sich und ihre Tochter mit dem MottoShirt von Wildfang

Quelle: https://www.instagram.com/p/BvFSGzzgU5p/

Hier existiert also von vornherein eine größere Bandbreite, die von denjenigen, die sich ein solches Produkt ausdenken, sicher schon von vornherein angelegt ist.

Eine solche Marke kann feministische Werte glaubwürdiger vertreten. Der Instagram-Account von Wildfang selbst vermittelt sogar den Eindruck, man sei auf einer Seite von politischen Aktivisten gelandet. Hier geht es ganz klar um ein politisches Programm, und $\mathrm{Zu}^{7}$ sehen ist etwa eine Parodie auf einen be- 
rühmten Mantel von Melania Trump, mit dem sie zu einem humanitären Termin fuhr, obwohl auf ihm der Slogan „I really don`t care, do you?“ steht. Das wurde von Wildfang umgekehrt in „I really care, don`t you?“, und eine Mitarbeiterin hat sich vor den Trump Tower gestellt, um das entsprechend zu inszenieren.

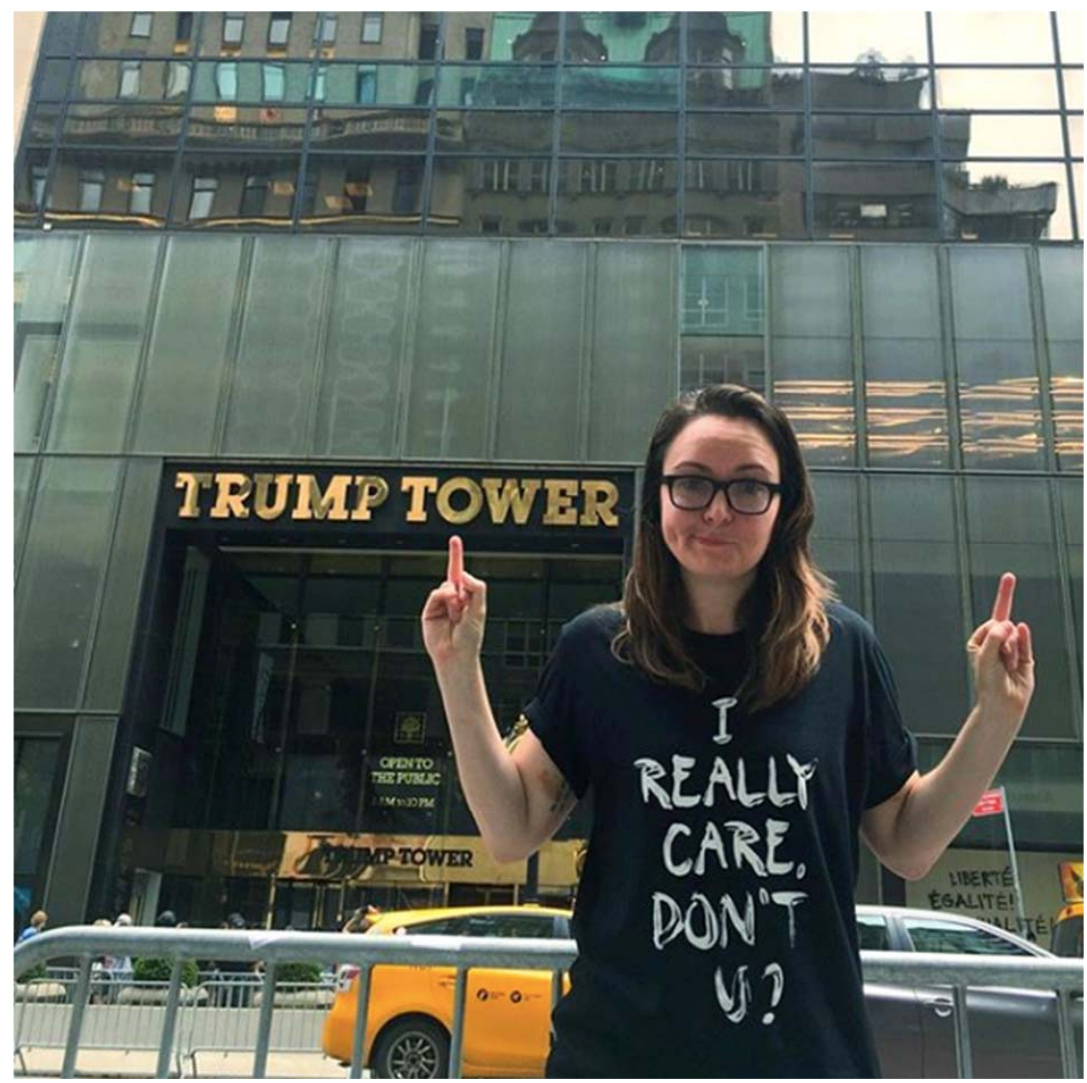

Abb. 3: Auf dem Instagram-Account von Wildfang wird gegen Melania Trump demonstriert

Quelle: https://www.instagram.com/p/BpA2qcbjQHC/ 


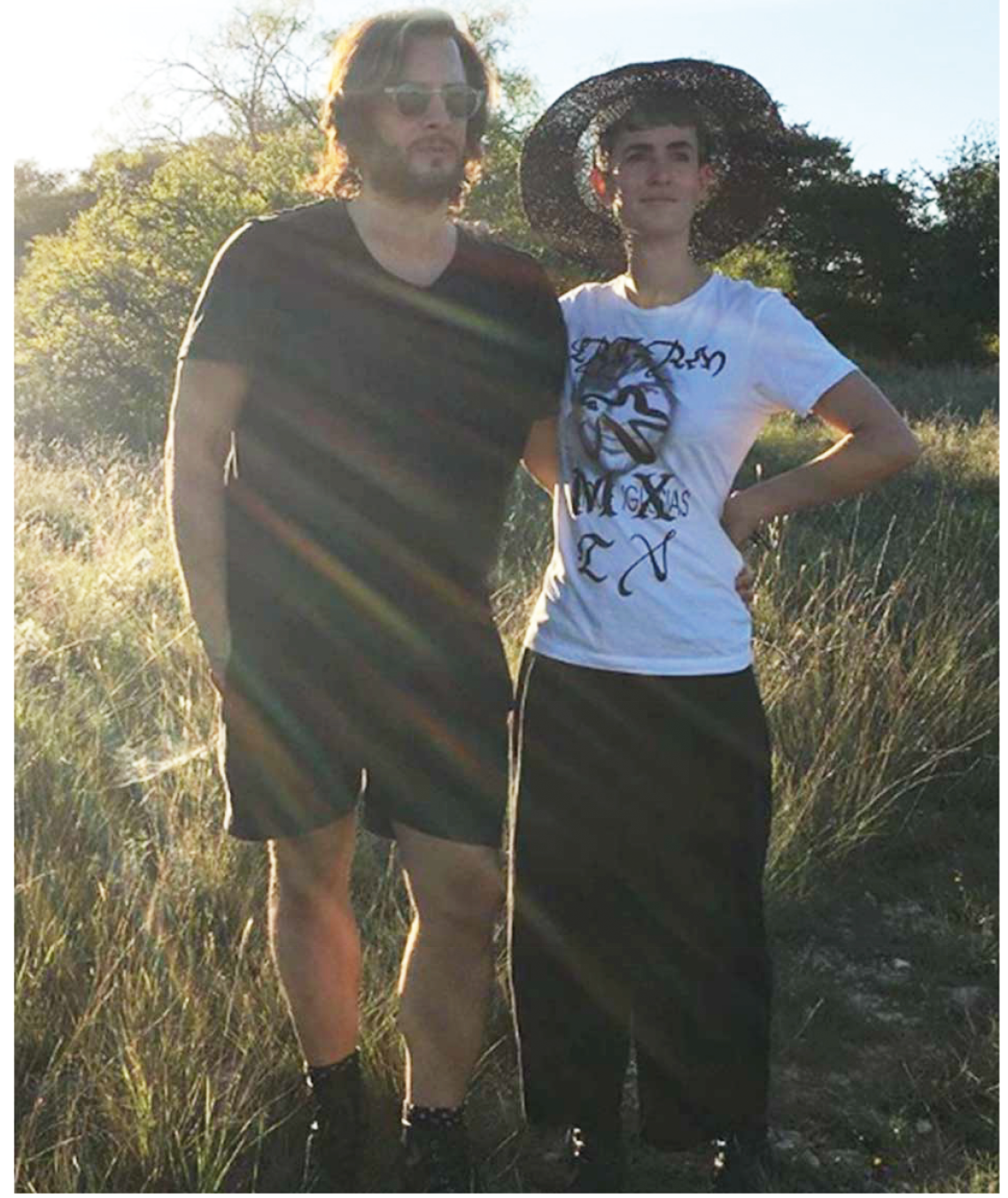

Abb. 4: Eine Konsumentin zeigt sich mit einem T-Shirt von Pia Camil

Quelle: https://www.instagram.com/p/Bbs4TmiBU4l/ 
Strategien des politischen Aktivismus werden also von einem Modelabel aufgegriffen, das aber zugleich ökonomischen Erfolg haben will. Statt (wie Dior) etwas Exklusives zu machen, will Wildfang zeigen, welche Power der Feminismus besitzt und dass wirklich ein gesellschaftlicher Wandel möglich ist. Dagegen gibt es viele andere Projekte, die den Konsumenten Exklusivität und damit zugleich ein Mehr an Individualität versprechen. Gerne wird etwa mit ,Limited Editions' gearbeitet - so etwa bei einer Zusammenarbeit der mexikanischen Künstlerin Pia Camil mit dem englischen Modedesigner Arren Louis, die 2016 Oberteile in einer Auflage von fünfzig Stück produzierten, von denen jedes anders aussieht. Aber auch hier geht es um gesellschaftspolitische Anliegen, da die dafür verwendeten Stoffe Ausschussware von Firmen in Mexiko waren. So wird eine Form von Upcycling betrieben, zugleich aber auf Wirtschaftskreisläufe, auf den Umgang mit Materialien, auf die Möglichkeiten von Nachhaltigkeit aufmerksam gemacht.

Doch wird die Exklusivität zusätzlich durch die Art der Codierung gesteigert. Als Künstlerin nähert Camil ihre Kleidungsstücke bei vielen ihrer Projekte nämlich Werken moderner Kunst an, sodass man oft gar nicht ohne weiteres verstehen kann, worin genau die Botschaft besteht. Wer ein solches Stück mit Geheimcode trägt, darf sich umso mehr als Elite oder als Insider fühlen, doch geht diese Coolness zugleich auf Kosten der Wirksamkeit der Botschaft. So etwa bei T-Shirts, die ursprünglich aus Lateinamerika stammen, dann in die USA verkauft wurden, um dort in Altkleidersammlungen zu gelangen und schließlich wieder in Mexiko zu landen. Dort hat Camil sie aufgekauft und erneut in die USA gebracht - nun als ,Limited Edition' und mit einem Aufdruck versehen, der diese Handelswege beschreiben soll, aber aus ziemlich rätselhaften Zeichen besteht.

In den letzten Jahren wurden etliche Unternehmen gegründet, die sich von vornherein auf Produkte spezialisieren, welche ästhetisch und moralisch höchsten Ansprüchen genügen. Sie haben in Concept Stores und Museumsstores ihren Auftritt, sind aber auch online präsent. Eines dieser Unternehmen heißt Forty Five Ten, ein anderes Third Drawer Down. Ein typisches Produkt eines solchen Unternehmens sind etwa Blumentöpfe von Group Partner, einer Töpferei aus Brooklyn. Sie sind fotogen, weil sie mit teils nackten, teils spärlich bekleideten Körpern ein erotisches Sujet haben, zugleich aber, nicht zuletzt wegen der Art ihrer Bemalung, witzig wirken. Man kann sie vielfältig inszenieren, dabei aber nicht nur Humor und Freizügigkeit beweisen, sondern zudem ein Bekenntnis zu einer multikulturellen Gesellschaft ablegen. Da sie aus verschiedenfarbigen Tonmassen geformt sind, stehen die Körper, die sie jeweils repräsentieren, nämlich für unterschiedliche Ethnien, was auf der Website des Unternehmens auch eigens vermerkt ist. 


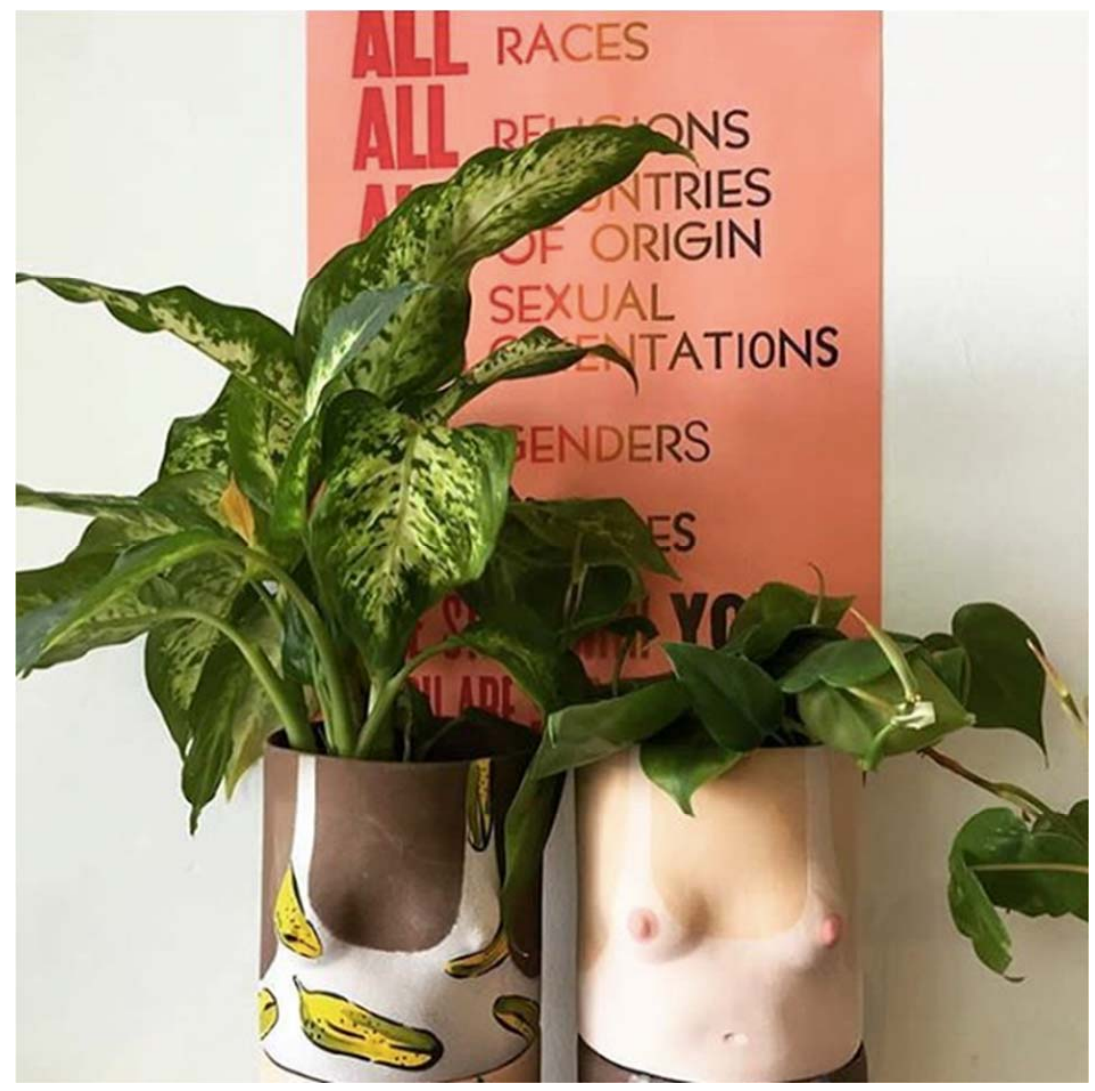

Abb. 5: Group Partner stellt auf dem eigenen Instagram-Account Blumentöpfe vor

Da das Material der Töpfe recycelbar ist, kann man mit ihnen schließlich sogar noch ökologische Sensibilität bekunden, die faktisch jedoch vermutlich dadurch konterkariert wird, dass sich der Gag, aus dem das Produkt besteht, relativ schnell erschöpft und selbst völlig intakte Töpfe deshalb schon bald gegen andere ausgetauscht werden. So fotogen Designgags sein mögen, so sehr beschleunigen sie also zugleich Konsumkreisläufe und sind damit ökologisch fragwürdig. Immerhin erlauben sie es, ökonomische Potenz zu zeigen, da die neuesten Gags nur um sich haben kann, wer sie sich auch leisten kann. 
Ein anderes Produkt, bei dem viele Aspekte idealtypisch zusammenkommen, ist eine Luftmatratze mit einem Motiv der Fotokünstlerin Cindy Sherman, hergestellt in nicht limitierter Auflage, für rund 300 Euro zu erwerben.

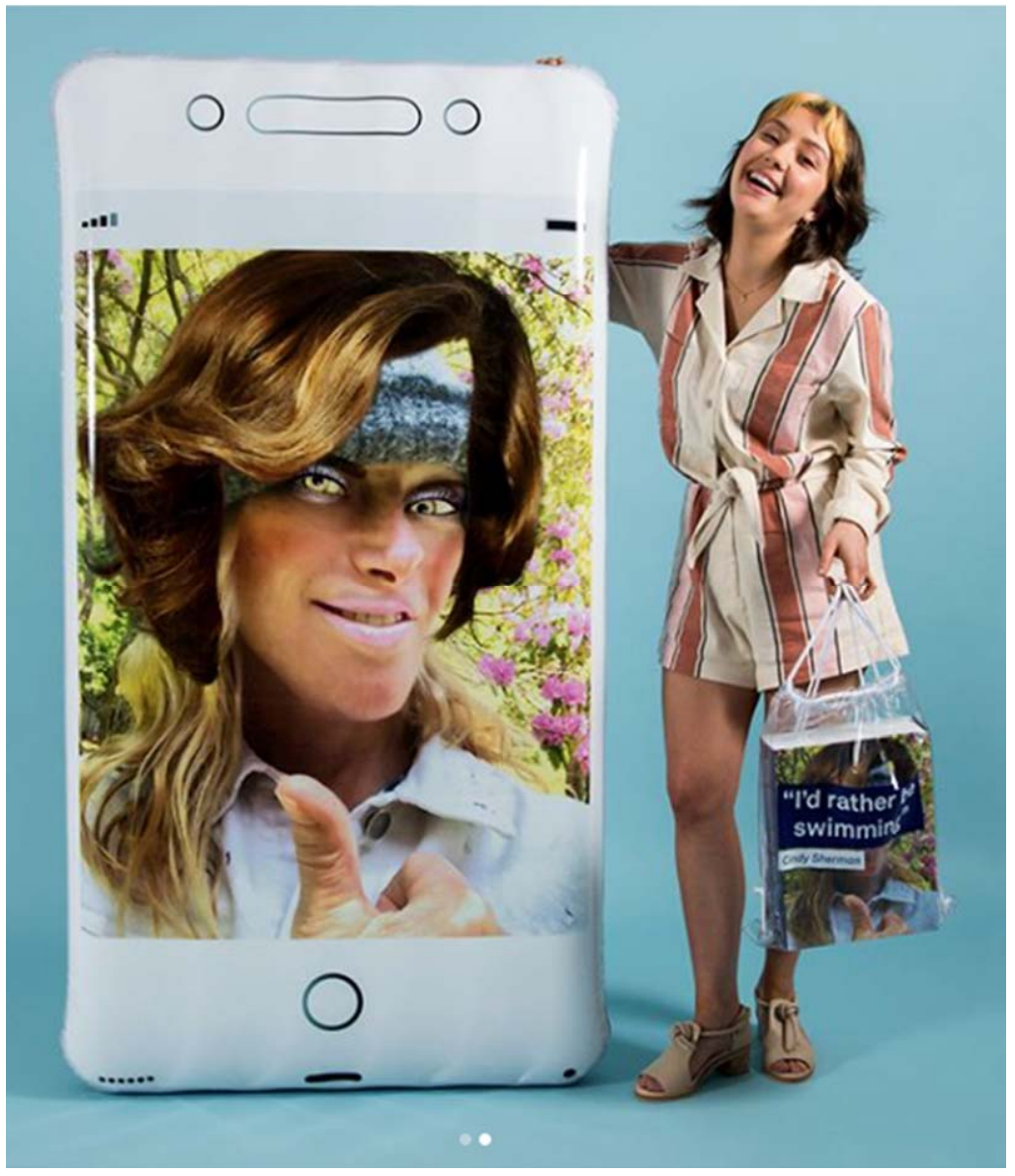

Abb. 6: Luftmatratze mit einem Motiv von Cindy Sherman auf dem InstagramAccount von Thid Drawer Down

Quelle: https://www.instagram.com/p/BkypfRBg700/ 
Das Motiv zeigt ein stark verfremdetes Selfie der Künstlerin, das sie ursprünglich auf ihrem Instagram-Account gepostet hatte und mit dem sie ihr eigenes früheres Werk des Sich-Maskierens und Sich-Deformierens unter den Bedingungen digitaler Bildkultur fortsetzt. Auf die Herkunft des Motivs wird bei der Luftmatratze auch angespielt, ist das Bild darauf doch in einem Rahmen zu sehen, der an ein Smartphone erinnert. So wird die (Selbst)inszenierung ausdrücklich zum Thema, und wenn ein Käufer einer Sherman-Matratze selbst wiederum ein Bild davon postet, schließt sich gleichsam der Kreis. Doch auch hier vollbringt man mit dem Kauf zugleich ein gutes Werk, weil ein Teil der Umsätze zur Finanzierung eines ökologischen Projekts verwendet wird, das die Wasserqualität im Hudson River in New York verbessern und den Bewohnern der Stadt ein sauberes, sicheres Schwimmbecken im Fluss zur Verfügung stellen soll.

Doch sosehr man sich mit Objekten wie der Luftmatratze als ästhetisch wie auch gesellschaftspolitisch versiert, also als rundum souveräner Konsumbürger zeigen kann, so sehr sind mit einem derartig demonstrativen Konsum problematische Konsequenzen verbunden. Zwei seien abschließend angesprochen.

Da mittlerweile so viele Möglichkeiten zum Konsum von Moral existieren, häufen manche Menschen geradezu einen Gewissenswohlstand an. Damit aber können sie Anwandlungen von Selbstgerechtigkeit und Hochmut kaum noch entkommen, es also nur schwerlich vermeiden, auf Menschen mit anderen Konsumgewohnheiten herabzublicken. Sosehr sie sich im Zuge ihres bekenntnishaften Konsums für Minderheiten und sozial Benachteiligte einsetzen mögen, so wenig reflektieren sie dann, welche sozialen Konsequenzen derselbe Konsum in ihrer eigenen Umgebung haben kann. Statt nämlich zu überlegen, woran es liegt, dass andere vielleicht nur deshalb nicht denselben Konsumstil pflegen, weil sie weniger Geld, weniger Bildung oder weniger Zeit, also schlechtere Voraussetzungen dafür haben, unterstellen sie ihnen mangelndes Wertbewusstsein. Diejenigen, die am heutigen Bekenntniswettbewerb nicht oder nur beschränkt teilnehmen können, erfahren daher eine pauschale Abwertung als moralische Personen. In einer Gesellschaft, in der Konsumprodukte zu einem Hauptmedium gerade auch moralischer Qualifikation geworden sind, findet somit in großem Umfang Exklusion und Klassenkampf statt. Eine fortschrittliche Sozialpolitik hätte genau da anzusetzen - und könnte dann eine gefährliche neue Klassengesellschaft verhindern.

Ein weiteres Problem wird sichtbar, wenn man überlegt, wie viel materiellen Aufwand die Produkte beanspruchen, die als Medien des Selbstausdrucks fungieren. Wer Werte mit Blumentöpfen, Luftmatratzen oder T-Shirts manifestiert, wird vieles nicht nutzen, bis es kaputt ist, sondern nur solange, bis einer anderen Überzeugung Ausdruck verliehen werden soll oder bis etwas gefunden ist, das dieselbe Aussage/ noch/aktueller, noch prägnanter, noch witziger, noch 
stilvoller zur Geltung bringt oder sich noch besser inszenieren lässt. Also kauft man eine neue Luftmatratze, weil sie ein schickes Sammlerstück ist oder sich ein guter Zweck damit verbinden lässt, aber nicht, weil man sie unbedingt braucht. Es ist also ziemlich inkonsequent, gerade Bekenntnisse zu einem ökologischeren oder sozialeren Lebensstil so kundzutun, dass dafür besonders viele Ressourcen verwendet werden müssen. Also sollte sich auch eine fortschrittliche Ökologiepolitik stärker einmischen, und man sollte sich angewöhnen, Bekenntnisse danach zu beurteilen, wie viele Ressourcen sie benötigen. Könnte man nicht auch das Foto einer Spendenquittung statt das einer Luftmatratze posten, um zu zeigen, dass man sich für die Reinigung des Hudson River einsetzt? Und würden nicht gerade diejenigen, die eine Luftmatratze nur kaufen, um sich moralisch zu profilieren, auf einmal als unverantwortlich gelten?

\section{Literatur}

Moser, S., Schlechtriemen, T. (2018): Sozialfiguren - zwischen gesellschaftlicher Erfahrung und soziologischer Diagnose. In: Zeitschrift für Soziologie 47/3, 164-180.

Ullrich, W. (2018): Konsum als Arbeit. In: Liessmann, K.P.: Mut zur Faulheit. Die Arbeit und ihr Schicksal, Paul Zsolnay Wien, 225-246. 
